
\title{
25 Research Square \\ Effect of cervical curvature reduction after ACCF on adjacent segmental lesions
}

\section{Lifeng Li}

The Third Xiangya Hospital,Central South University

$\mathrm{Na} \mathrm{Li}$

The Third Xiangya Hospital,Central South University

Jiahui Zhou

The Third Xiangya Hospital,Central South University

Handong Li

The Third Xiangya Hospital,Central South University

\section{Xianping Du}

Embry-Riddle Aeronautical University

Haibo He

The Third Xiangya Hospital,Central South University

\section{Pengfei Rong}

The Third Xiangya Hospital,Central South University

\section{Yin Liu ( $\sim$ jyy796@163.com )}

The Third Xiangya Hospital Central South University https://orcid.org/0000-0002-7116-8383

\section{Wei Wang}

The Third Xiangya Hospital,Central South University

\section{Research article}

Keywords: Vertebral resection, Fusion, Adjacent segmental lesions, Cervical curvature, Cervical spondylotic myelopathy

Posted Date: August 15th, 2019

DOI: https://doi.org/10.21203/rs.2.12934/v1

License: (9) This work is licensed under a Creative Commons Attribution 4.0 International License. Read Full License 


\section{Abstract}

Objective: To investigate the relationship of adjacent segment degeneration (RASP) and the variation of cervical curvature and vertebral height after Anterior Cervical Corpectomy and Fusion (ACCF). Methods: A retrospective analysis of 80 patients with cervical spondylotic myelopathy (CSM) who had been followed up for at least 1 year after ACCF. The patients were divided into RASP group and no RASP group according to whether the RASP occured after surgery. On the standing cervical lateral radiograph of all patients before and after surgery at the short-term and final follow-up, the C2-7 Cobb angle of the cervical vertebrae, the Cobb angle of the surgical segment and the height of the fusion segment for the anterior and posterior segments of the vertebral body were measured. The difference between the two groups of patients before and after surgery was calculated, as well as its correlation with the occurrence of RASP. Result: there were 37 patients in the RASP group and 43 patients in the no-RASP group. In RASP group, the short-term average curvature of cervical vertebra was significantly reduced after surgery. However, there was no significant difference in no RASP group. The height of the anterior and posterior fusion segments in two groups was significantly higher than that before surgery, however, which was significantly lower during the final follow-up than that in the short-term after surgery. Cox regression analysis showed that only the variation of postoperative C2-7Cobb angle was associated with RASP. Conclusion: The reduction of cervical curvature after ACF may be related to postoperative RASP. Maintaining good cervical curvature after operation may be expected to reduce the occurrence of RASP after ACF.

\section{Introduction}

Timely surgery can effectively improve the clinical symptoms and prognosis of patients with cervical spondylotic myelopathy (CSM). The clinical effect of anterior cervical corpectomy and fusion (ACCF) in the treatment of CSM has been repeatedly demonstrated [1-3]. With the continuous improvement of modern medical technology, the long-term efficacy of the surgery for cervical spondylosis attracts more and more attention. Adjacent segmental lesion is one of the important complications that affects the long-term efficacy of cervical fusion and the 10-year incidence is about $25 \%$ [4], and the adjacent segmental lesions occur more frequently on imaging [5-7]. At present, the causes and mechanisms of adjacent segmental lesions are still unclear.

Good cervical fusion after surgery is the key factor to maintain the physiological structure and function of the cervical spine. In this process, it is influenced by the physiological gravity and often accompanied by the implantation of the implant into the adjacent vertebral body. Therefore, it may cause changes in the structure and curvature of the cervical spine after surgery. However, the effects of this change on cervical curvature and long-term clinical outcome after ACCF remain controversial [8-11].

To further clarify the occurrence of adjacent segmental lesions after ACCF and the effects of surgical segment height or cervical curvature on adjacent segmental lesions, we measured the height of fusion segment, cervical curvature, postoperative clinical recovery and the occurrence of adjacent segmental lesions of 80 patients at least 1 year after ACCF. Moreover, we analyzed the correlation between these factors.

\section{Information And Methods}

\subsection{Research object}


A retrospective analysis was performed on 80 patients with CSM who underwent ACCF surgery in the Department of Medical Imaging in our hospital during 2008.1.1-2013.12.31. The inclusion criteria were the patients who got anterior cervical fusion for cervical spondylosis, with a minimum follow-up of 1 year and having complete imaging and clinical data. Exclusion criteria included: 1. Patients who had undergone cervical surgery or combined with posterior cervical surgery; 2 . Combined with other cervical non-degeneration diseases such as fractures, active infections or inflammation, the diseases of connective tissue, tumors, or congenital dysplasia. There were 37 males and 43 females with an average age of $50.5 \pm 8.9$ years during the operation, ranging from 29 to 75 years old. All patients received their written consent or telephone verbal consent. Cervical surgery in all patients was performed in our hospital. The procedures were partial vertebral body resection and titanium cage autologous bone implantation and plate fixation of anterior cervical spine. The mean follow-up duration was $29.8 \pm 18.1$ months, ranging from 12 to 88 months.

\subsection{Test indicators and methods}

The imaging data of all patients before and after surgery (within 2-4 weeks after surgery) and at the final follow-up (at least 1 year after surgery) were analyzed at the PACS workstation. The imaging examination data included positive and lateral radiographs of the cervical vertebra, CT and/or MRI data of the cervical vertebrae for preoperative operation and final follow-up. All inspections are performed in the department according to the same standard process. If the patient has a new or aggravated degeneration changes in the adjacent segment, it is diagnosed as RASP, such as narrowing of the intervertebral space, enlargement of the osteophytes in vertebral body, endplate sclerosis, uncinate hyperplasia, facet joint degeneration, etc. [12]. The C2-7 Cobb angle, the Cobb angle of the surgical segment, and the height of the anterior and posterior of the fusion segment were measured by standing lateral radiographs of the cervical spine before and after the surgery on short-term and final follow-up for each patient (Figure 1). The indicators were measured twice by two senior imaging diagnostic doctors during one month, and the average was taken for data analysis. The diagnosis of RASP for each patient was recognized by two doctors.

\subsection{Preoperative and postoperative clinical conditions}

The severity of cervical spondylosis was evaluated by JOA score before, after the surgery, as well as the final follow-up. The surgical outcome was measured by the Recovery Rate (RR). RR was calculated by JOA scores in preoperative and early postoperative time (in one month). The specific formula is as follows: RR (\%) = (postoperative JOA score - preoperative JOA score) / (normal score (17) - preoperative JOA Score $) \times 100[13]$

\subsection{Statistical analysis}

All statistical analyses were calculated by SPSS 18.0 software. To compare the clinical and imaging characteristics of the two groups of patients, continuous variables were analyzed using paired t-test or Mann-Whitney U test. The count data were analyzed by Fisher's or chi-square test. Single factor 
correlation was analyzed using Pearson correlation analysis. The factors with $p<0.05$ were further analyzed using Cox regression to analyze the predictive value of ASD. $p$ value $<0.05$ was considered statistically significant.

\section{Result}

Except for the length of follow-up, clinical features of the patients, such as surgical site, number of fusion segments, and JOA scores for preoperative, postoperative, and final follow-up, had no significant difference in RASP group and no RASP group, (Table 1). The JOA scores in short-term after surgery for all patients were significantly higher than those before surgery. The JOA score of the final follow-up of the RASP group was significantly lower than that of the postoperative in short-term time, but the RASP-free group was significantly higher than the short-term after surgery. In RASP group, the JOA score of the last follow-up was significantly lower than that of short-term after surgery.

The changes of the anterior and posterior heights of the surgical fusion segment, the Cobb angle of the cervical C2-7 of the surgical fusion segment at three time points before and after surgery was shown in Table 2. Compared with the preoperative situation, the height of the anterior and posterior fusion segments of the two groups was significantly increased, however, which was significantly lower at the final follow-up than that in short-term after surgery. The short-term C2-7 Cobb angle in the RASP group was significantly lower than that before surgery, and the C2-7 Cobb angle at the final follow-up was increased, but still significantly lower than that after surgery. In no RASP group, C2-7 and the Cobb angles of fusion segments of the patients were significantly higher in the short-term and final follow-up than that before surgery.

The correlation between the imaging parameters and the occurrence of RASP was analyzed and the results demonstrated that in the short-term of the surgery, the change of anterior height of fusion segment was significantly correlated with that of posterior height (correlation coefficient was 0.599 , $p<0.001)$. In the short-term of the surgery, the height change of anterior fusion segment was significantly correlated with C2-7Cobb angle change in the short-term of the surgery (correlation coefficient of 0.274 , $p=0.010$ ) and Cobb angle change of fusion segment (correlation coefficient of $0.333, p=0.002$ ). C2-7 Cobb angle and fusion section Cobb angle were both related to RASP. After adjusting for the patient's age, gender, surgical site and surgical segment, etc. COX regression analysis was further performed and the result showed that only $\mathrm{C} 2-7 \mathrm{Cobb}$ angle change was significantly correlated with the occurrence of RASP and had predictive value, with a hazard ratio (HR) of 0.454 (Table 3).

\section{Discussion}

A large number of clinical studies have proved that ACF surgery can effectively relieve the pressure of the neck nerves, stabilize or alleviate the symptoms caused by cervical spondylosis, and with the younger and younger patients undergoing surgery, the long-term efficacy of surgery and the quality of life of patients The impact of this study is receiving more and more attention. Among them, the study of 
adjacent segmental lesions (ASP) after ACF is one of the current hotspots, and it has become one of the main reasons for reoperation after ACCF surgery [4].

Adjacent segmental imaging lesions after cervical fusion is one of the manifestations of ASP, which was mainly diagnosed by imaging examination with the objective and quantifiable characteristics. In the present study, among the 80 patients included, the incidence of RASP was $46.25 \%$, which was basically consistent with previous studies. The report was similar to previous reports [5-7], although there was no significant difference in short-term recovery rates between the two groups. The mean recovery rate at the final follow-up was significantly lower in the RASP group than that in the no-RASP group, as well as the higher proportion of symptoms in RASP group with severe imaging changes than that in no RASP group. However, the correlation between RASP and CASP is still controversial, and some researchers believe that the correlation between RASP and CASP is small or completely irrelevant [8-9]. In RASP group, there was no significant correlation between the occurrence of RASP and the deterioration of clinical symptoms of patients. This might be due to the relatively short follow-up period in RASP group, in which the number of CASP or reoperation is small, which affected the results of statistical analysis of this study.

At present, the influence of height change of cervical fusion segment on postoperative cervical curvature or RASP was still unclear. The results of this study found that most of the titanium cages implanted during ACCF surgery showed different degrees of collapse [15-17], in which the type of bone graft, titanium cage diameter and placement site may be related to it; Jang et al [16] gave an retrospective analysis of 30 patients with cervical spondylosis with ACCF and titanium cage implantation, $93.3 \%$ of the implants were depressed, and the posterior implant was more obvious. However, the cervical and segmental curvature were both increased at the final follow-up. The use of the pre-steel plate may also affect the curvature of the cervical spine after ACDF. All the patients included in this study were partial vertebral body resection and titanium cage implantation. The trend of height change of fusion segment was similar to that of Jang et al. Both of the results showed postoperative increase in short-term and a reduction of the final follow-up. This height of the vertebral body may be related to the change of cervical curvature after ACF, but Cox regression analysis showed that only the change of cervical curvature was significantly related to the occurrence of RASP, and the change of vertebral height was not related to the occurrence of RASP. This result suggested that changes in the vertebral height of the surgical segment after ACCF did not cause cervical vertebrae alignment or stability abnormalities, which may had no effect on adjacent segments.

The mechanism of ASP after anterior cervical fusion was still unclarified. In vivo and in vitro studies have found that the range of motion of adjacent segments after fusion was significantly increased, and the intravertebral disc pressure was significantly increased. Therefore, the changes of the biomechanics of adjacent segments of cervical spine surgery may be related to ASP. Compared with other parts of the spine, the cervical vertebra had its unique morphological characteristics and highly complex anatomical structure. The normal anterior curvature of the cervical vertebra was one of the important morphological features of the cervical vertebrae, which helped to maintain the normal distribution of cervical biomechanical load, among of this, $36 \%$ of which was distributed. In the anterior column, the middle 
posterior column (bilateral facet joints and articular processes) was subjected to $64 \%$ of the force [18]. Once the cervical curvature changes, it may cause differences in the biomechanical distribution of the cervical vertebrae, which may also be positive, which might be the mechanism that affected the occurrence of ASP, but this speculation remained to be confirmed. Park et al [19] also found that no matter discectomy or vertebral resection, cervical curvature may decrease after surgery, but this situation mainly occurred within 6 weeks after surgery; after 6 weeks after surgery, the degree of reduction gradually improved, but the research did not show the comparison with the preoperative angle. In our study, we also found that postoperative curvature of the majority of the patients showed decreased compared with their preoperative curvature, however, in RASP group, the cervical C2-7 curvature at the final follow-up was still less than that of the preoperative situation, and the situation was converse in non-RASP group to RASP group.

There were limitations and deficiencies in this study. First of all, as this study was retrospective, the sample size was relatively small, the data was heterogeneous, and the influence of sampling error or recall error cannot be excluded. However, there was no significant difference between the confounders of the two groups, so I believed the effect was not sufficient to cover up the difference between the two groups and should not affect the accuracy of the final result, but it did not rule out the extent of the extrapolation that may affect the data to some extent. Thus, the prospective study with better data homogeneity need further to be confirmed. Secondly, this study only had a research on the correlation between cervical Cobb angle and RASP. However, because the data included in this group were retrospective data, other important cervical alignment parameters, such as sagittal vertical wheelbase (SVA) and vertical angle (CBVA) of the mandibular-bow arch could not be studied, so further prospective studies were still needed to be confirmed.

Therefore, the reduction of cervical spine curvature after ACCF surgery may be related to the occurrence of postoperative RASP. The changes of anterior height of the surgical segment may be related to the changes of cervical curvature after operation. It may be expected to reduce the occurrence of adjacent segmental degeneration after ACCF to maintain good cervical curvature after operation, at least not less than the preoperative cervical curvature

\section{Declarations}

Acknowledgements

We are grateful to all doctors in the Department of Radiology and Orthopaedics of the Third Xiangya Hospital.They provide care and research support.

\section{AVAILABILITY OF DATA AND MATERIALS}

The datasets used or analysed during the current study are available from the corresponding author on reasonable request. 
Funding

The following support was obtained in this study.

1. Natural Science Foundation of Hunan Province(2018JJ3799);

2. the New Xiangya Talent Project of the Third Xiangya Hospital of Central South University (JY201712)

Compliance with ethical standards

Ethical approval

Patients who participated in the study were informed by SMS or by phone, and were ethically approved by the Research Ethics Committee of the Third Xiangya Hospital of Central South University.

Conflicts of interest

The authors declare that they have no conflicts of interest.

\section{References}

[1]. Lu T, Liu C, Yang B, Liu J, Zhang F, Wang D, Li H, He X. Single-Level Anterior Cervical Corpectomy and Fusion Using a New 3D-Printed Anatomy-Adaptive Titanium Mesh Cage for Treatment of Cervical Spondylotic Myelopathy and Ossification of the Posterior Longitudinal Ligament: A Retrospective Case Series Study. Med Sci Monit 2017;23:3105-3114.

[2]. Schulz C, Mauer U M, Mathieu R. [Clinical and Radiological Results after Anterior Cervical Corpectomy with Cage Fusion - a Retrospective Comparison of PEEK vs. Titanium Cages]. Z Orthop Unfall 2017;155:201-208.

[3]. Ghogawala Z. Anterior Cervical Option to Manage Degenerative Cervical Myelopathy. Neurosurg Clin N Am 2018; 29:83-89.

[4]. Hilibrand AS, Carlson GD, Palumbo MA, Jones PK, Bohlman HH. Radiculopathy and Myelopathy at Segments Adjacent to the Site of a Previous Anterior Cervical Arthrodesis. J Bone Joint Surg Am 1999;81:519-528.

[5]. Goffin J, Geusens E, Vantomme N, Quintens E, Waerzeggers Y, Depreitere B, Van Calenbergh F, van Loon J. Long-term follow-up after interbody fusion of the cervical spine. J Spinal Disord Tech 2004;17:79-85.

[6]. Ikenaga M, Shikata J, Tanaka C. Long-term results over 10 years of anterior corpectomy and fusion for multilevel cervical myelopathy. Spine 2006; 31:1568-1574. 
[7]. Bydon M, Xu R, De G R, et al. Adjacent segment disease after anterior cervical discectomy and fusion: Incidence and clinical outcomes of patients requiring anterior versus posterior repeat cervical fusion. Surg Neurol Int 2014;5:S74-S78.

[8]. Ishihara $\mathrm{H}$, Kanamori M, Kawaguchi $\mathrm{Y}$, Nakamura H, Kimura T. Adjacent segment disease after anterior cervical interbody fusion. Spine J 2004;4:624-8.

[9]. Matsumoto M, Okada E, Ichihara D, Watanabe K, Chiba K, Toyama Y, Fujiwara H, Momoshima S, Nishiwaki Y, Iwanami A, Ikegami T, Takahata T, Hashimoto T. Anterior cervical decompression and fusion accelerates adjacent segment degeneration: comparison with asymptomatic volunteers in a ten-year magnetic resonance imaging follow-up study. Spine 2010;35:36-43.

[10]. Kawakami M, Tamaki T, Ando M, Yamada H, Yoshida M. Relationships between sagittal alignment of the cervical spine and morphology of the spinal cord and clinical outcomes in patients with cervical spondylotic myelopathy treated with expansive laminoplasty.J Spinal Disord Tech 2002;15:391-7.

[11]. Amandeep B, Rolfe K W. Inadequate Surgical Decompression in Patients with Cervical Myelopathy: A Retrospective Review. Global Spine J 2016;6:542-547.

[12]. Park JB, Cho YS, Riew KD. Development of adjacent-level ossification in patients with an anterior cervical plate. J Bone Joint Surg Am 2005;87:558-63.

[13]. Odate S, Shikata J, Soeda T, Yamamura S, Kawaguchi S. Surgical results and complications of anterior decompression and fusion as a revision surgery after initial posterior surgery for cervical myelopathy due to ossification of the posterior longitudinal ligament. J Neurosurg Spine 2017; 26:466473.

[14]. Ding C, Cicuttini F, Jones G. How important is MRI for detecting early osteoarthritis?. Nat Clin Pract Rheumatol 2008;4:4-5.

[15]. Chen Y, Chen D, Guo Y, Wang X, Lu X, He Z,Yuan W. Subsidence of titanium mesh cage: a study based on 300 cases. J Spinal Disord Tech 2008;21:489-92.

[16]. Jang JW, Lee JK, Lee JH, Hur H, Kim TW, Kim SH. Effect of posterior subsidence on cervical alignment after anterior cervical corpectomy and reconstruction using titanium mesh cages in degenerative cervical disease. J Clin Neurosci 2014;21:1779-85.

[17]. MA Yonggang $\triangle L I U$ Shiqing $\triangle L I$ Yaming. Titanium cages subsidence following anterior cervical decompression and fusion.Chinese Journal of Spine and Spinal Cord 2011;21:21-23.

[18].Pal GP, Sherk HH. The vertical stability of the cervical spine. Spine 1988;13:447-9.

[19]. Park MS, Kelly MP, Lee DH, Min WK, Rahman RK, Riew KD. Sagittal alignment as a predictor of clinical adjacent segment pathology requiring surgery after anterior cervical arthrodesis.Spine $\mathrm{J}$ 


\section{Tables}

Table 1. Comparison of general information and clinical information between patients of RASP and without RASP

\begin{tabular}{|c|c|c|c|}
\hline & RASP group $(\mathrm{N}=37)$ & $\begin{array}{c}\text { no RASP group } \\
(\mathrm{N}=43)\end{array}$ & $p$ value \\
\hline age $\left(\right.$ years old) ${ }^{\S}$ & $50.3(9.3)$ & $51.0(8.7)$ & 0.87 \\
\hline Gender (number of males) & 18 & 19 & 1.00 \\
\hline Vertebrectomy site & 2 & 5 & \\
\hline $\mathrm{C} 4$ & 17 & 18 & 0.55 \\
\hline C6 & 2 & $3^{12}$ & 0.48 \\
\hline $\begin{array}{l}\text { C4-5 } \\
\text { C5-6 }\end{array}$ & 3 & 5 & $\begin{array}{l}0.86 \\
0.88\end{array}$ \\
\hline Length of follow-up (months) ${ }^{\S}$ & $36.8(20.7)$ & $23.7(12.9)$ & $<0.01$ \\
\hline Preoperative JOA score ${ }^{\S}$ & $10.48 \square 2.79 \square$ & $10.78 \square 2.85 \square$ & 0.82 \\
\hline Postoperative JOA score ${ }^{\S}$ & $13.71 \square 2.24 \square^{*}$ & $14.42 \square 2.44 \square^{*}$ & 0.91 \\
\hline Follow-up JOA score ${ }^{\S}$ & $13.69 \square 2.20 \square^{* *}$ & $14.60 \square 2.58 \square^{* *}$ & 0.23 \\
\hline Postoperative recovery rate $(\% \square \S$ & $60.3 \square 29.8 \square$ & $61.6 \square 25.3 \square$ & 0.83 \\
\hline
\end{tabular}

$\S$ Mean (standard deviation) ${ }^{*}$ compared with pre-operation $\mathrm{p} \square 0.05{ }^{* *}$ compared with short-term after surgery $\mathrm{p} \square 0.05 \square$

Table 2. Comparison of preoperative and postoperative imaging parameters between RASP group and no RASP group

\begin{tabular}{|c|c|c|c|c|}
\hline & & RASP group & no RASP group & $\bar{P}$ value \\
\hline Before surgery & $\begin{array}{l}\mathrm{AFH} / \mathrm{cm} \\
\mathrm{PFH} / \mathrm{cm} \\
\mathrm{C} 27 \mathrm{CA} /{ }^{\circ} \\
\mathrm{FCA} /{ }^{\circ}\end{array}$ & $\begin{array}{l}5.22 \pm 1.23 \\
5.12 \pm 1.12 \\
20.42 \pm 9.92 \\
6.76 \pm 7.16\end{array}$ & $\begin{array}{l}5.27 \pm 1.15 \\
5.14 \pm 1.06 \\
12.65 \pm 11.91 \\
4.97 \pm 8.37\end{array}$ & $\begin{array}{l}0.86 \\
0.91 \\
<0.01 \\
0.29\end{array}$ \\
\hline Short-term after surgery & $\begin{array}{l}\mathrm{AFH} / \mathrm{cm} \\
\mathrm{PFH} / \mathrm{cm} \\
\mathrm{C}^{\circ} 7 \mathrm{CA} /^{\circ} \\
\mathrm{FCA}^{\circ}\end{array}$ & $\begin{array}{l}5.55 \pm 1.20^{* *} \\
5.38 \pm 1.20^{* *} \\
13.81 \pm 8.52^{* *} \\
7.12 \pm 5.47\end{array}$ & $\begin{array}{l}5.86 \pm 1.47^{* *} \\
5.63 \pm 1.40^{* *} \\
15.35 \pm 10.05^{*} \\
9.06 \pm 6.92^{* *}\end{array}$ & $\begin{array}{l}0.29 \\
0.37 \\
0.45 \\
0.15\end{array}$ \\
\hline The last follow-up & $\begin{array}{l}\mathrm{AFH} / \mathrm{cm} \\
\mathrm{PFH} / \mathrm{cm} \\
\mathrm{C} 27 \mathrm{CA} /{ }^{\circ} \\
\mathrm{FCA}^{\circ}\end{array}$ & $\begin{array}{l}5.19 \pm 1.09^{\S \S} \\
5.05 \pm 1.05^{\S \S} \\
17.02 \pm 9.67 \text { *§§§ } \\
6.38 \pm 6.26\end{array}$ & $\begin{array}{l}5.39 \pm 1.17^{* *} \square \S \S \\
5.14 \pm 1.06^{\S \S} \\
19.43 \pm 9.63^{* * \square \S \S} \\
9.45 \pm 7.52^{* *}\end{array}$ & $\begin{array}{l}0.42 \\
0.67 \\
0.25 \\
0.04\end{array}$ \\
\hline
\end{tabular}

${ }^{*}$ compared to pre-surgery $\mathrm{p} \square 0.05 \square^{* *}$ compared to pre-surgery $\mathrm{p} \square 0.01 \square \S_{\text {compared }}$ to post-

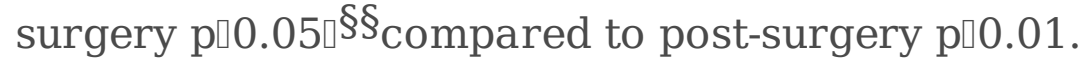


Table 3 Cox regression analysis of the correlation between cervical curvature changes and RASP

\begin{tabular}{ccccc}
\hline parameter & & & P value & HR \\
\hline C2-7 Cobb angle & -0.790 & 13.416 & $0.000^{*}$ & $0.454 \square 0.297-0.692 \square$ \\
Cobb angle of the fused segment & 0.090 & 3.313 & 0.069 & $1.094 \square 0.993-1.206 \square$ \\
The height of anterior fusion segment & 1.996 & 3.614 & 0.057 & $7.363 \square 0.940-57.665 \square$ \\
The height of posterior fusion segment -1.282 & 1.626 & 0.202 & $0.278 \square 0.039-1.990 \square$ \\
\hline
\end{tabular}

\section{Figures}




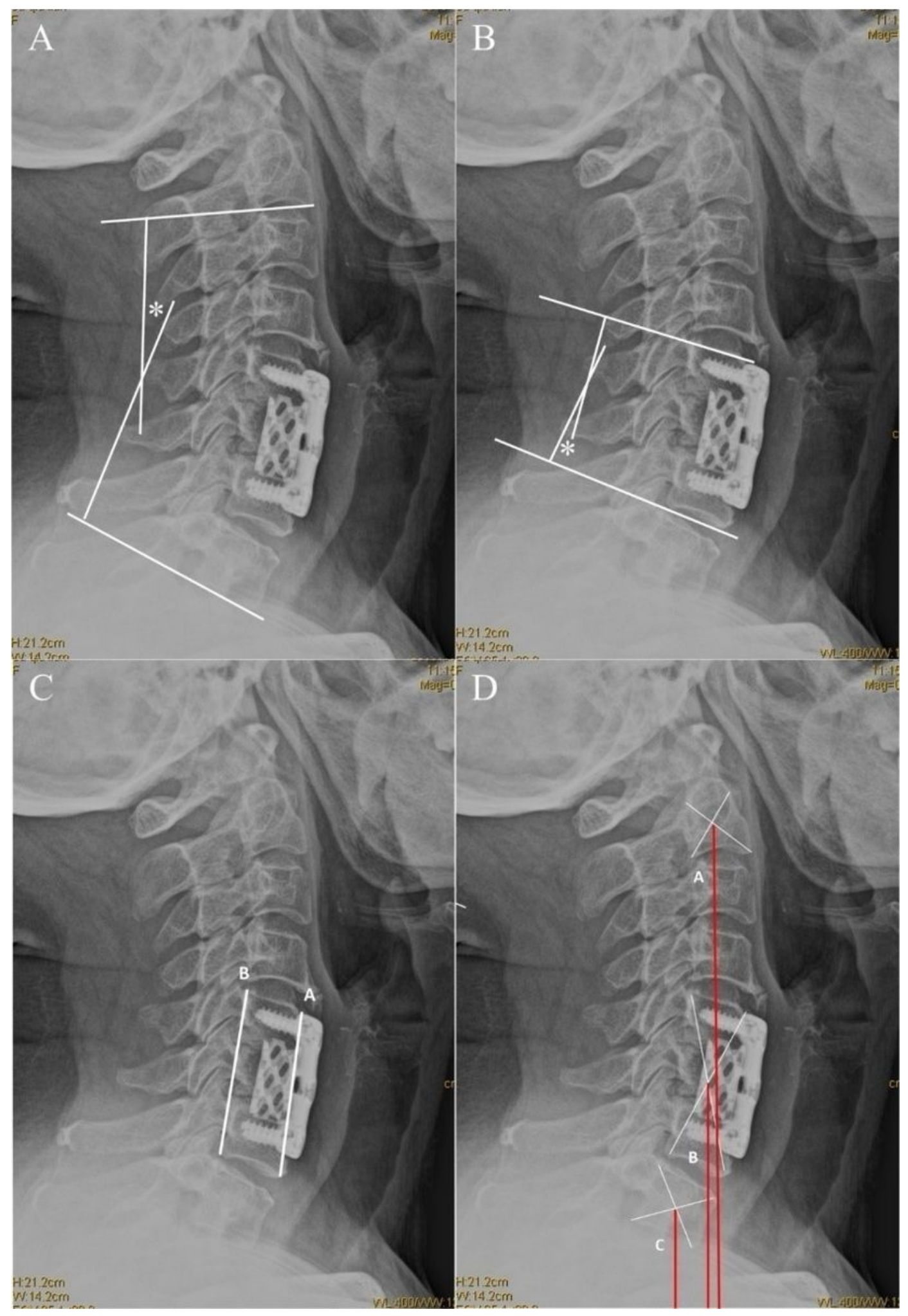

\section{Figure 1}

Imaging parameters of cervical vertebrae. A showed C2-7 Cobb angle measurement as indicated by *; B showed Cobb angle of fused segment as indicated by *; C showed the anterior (line A) and posterior (line B) height of fused segment. 